\title{
Analytical bioluminescence and chemiluminescence
}

\author{
Mara Mirasoli • Elisa Michelini
}

Published online: 11 July 2014

(C) Springer-Verlag Berlin Heidelberg 2014

Bioluminescence and chemiluminescence are well-established detection techniques with a wide range of applications in different fields from the life sciences to clinical, environmental, food, and pharmaceutical analysis. Such techniques have proved very powerful in terms of detectability, rapidity, and suitability for miniaturization and biosensor formats.

Although chemiluminescence and bioluminescence detection are characterized by low-intensity emissions, their main peculiarity is their high sensitivity, because of their very low nonspecific background signal and high signal-to-noise ratio. This, with their wide linear dynamic ranges and the possibility of detecting and quantifying light emissions down to a few photons by use of photomultiplier tubes, photodiodes, or such imaging systems as charge-coupled devices (CCD) or complementary metal-oxide semiconductor (CMOS) light sensors, make chemiluminescence and bioluminescence unique tools for development of ultrasensitive analytical and bioanalytical methods.

This special issue encompasses well-established applications (for example, liquid chromatography with chemiluminescence detection, reporter gene technology, in-vivo bioluminescence imaging) and recent trends and active research fields (for example, applications of nanomaterials and composite materials to modify and enhance the emission, and integration of bioluminescence, electrochemiluminescence and chemiluminescence-based detection in miniaturized analytical systems for point-of-care applications, lab-on-chip, microarrays, biosensors, and lab-on-paper based devices).

Since chemiluminescence was first introduced in the late 1970s its analytical performance has improved substantially

Published in the topical collection Analytical Bioluminescence and Chemiluminescence with guest editors Elisa Michelini and Mara Mirasoli.

M. Mirasoli $(\square) \cdot$ E. Michelini $(\triangle)$

Department of Chemistry, G. Ciamician, University of Bologna, Via

Selmi 2, 40126 Bologna, Italy

e-mail: mara.mirasoli@unibo.it

e-mail: elisa.michelini8@unibo.it and it is now extensively used as a detection principle in separative analytical techniques, enzyme label-based bioassays, for example enzyme immunoassays and nucleic acid assays, and in-vitro imaging. It also finds applications in several wellestablished clinical diagnostics assays. The growth in the number of publications worldwide on chemiluminescence- and electrochemiluminescence-based assays reflects the increased interest in this field. The papers in this special issue of Analytical and Bioanalytical Chemistry are indicative of the most recent and up-to-date analytical applications of chemiluminescence. Recent advances and current research are represented, including the development of chemiluminescence microfluidics and microarray-based assays, point-of-care and on-site test devices, paper-based analytical formats, and nanoparticle-enhanced chemiluminescence and electrogenerated chemiluminescence.

A new era in applied bioluminescence started with the first transgenic tobacco plant expressing the firefly luciferase gene, obtained approximately 30 years ago in Marlene Deluca's laboratory. Since then we have witnessed a revolution in basic and applied research, with new tools for investigating molecular targets at the cellular and subcellular level.

Several bioluminescent proteins have been isolated from a variety of luminescent marine and terrestrial organisms and the corresponding genes have been cloned. In addition, several luminescent proteins with improved spectral properties (e.g. with higher emission efficiency and different emission wavelengths) have been obtained by random or site-directed mutagenesis, enabling the development of highly sensitive and/or multiplexed assays.

Determining the natural mechanisms responsible for light emission has thus provided exciting new tools in the biosciences, and a large range of methods is now available for environmental, medical, and food analysis.

In fact, besides its spectacular aspects, bioluminescence is, with chemiluminescence, an extremely useful analytical technique enabling ultrasensitive detection in biotechnological applications and high-throughput screening. 
A large range of bioluminescent proteins and calciumdependent photoproteins, for example aequorin, is available for different bioanalytical applications, ranging from single-cell bioluminescence imaging to in-vivo whole-body imaging, from cell-based high-throughput drug screening to calcium monitoring. This collection of papers reports a selection of bioanalytical assays based on bioluminescent proteins, including immunoassays, protein-protein and protein-ligand interaction assays, whole-cell bioassays, and bioluminescence in-vivo imaging.

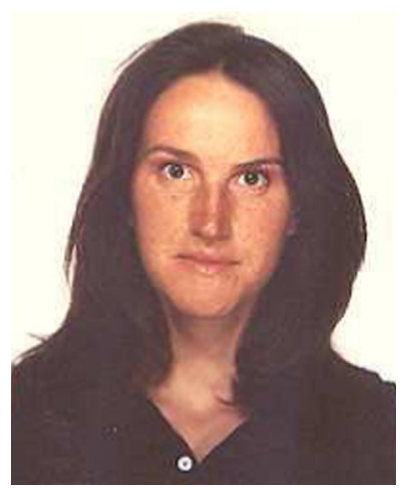

Mara Mirasoli is a researcher in analytical chemistry at the Alma Mater Studiorum, University of Bologna. Her research interests focus on the development and optimization of multiplex bioanalytical assays based on chemiluminescence detection, for example enzyme-based assays, immunoassays and gene-probe hybridization assays with applications in clinical, environmental, and food analysis. Most recently she has been working on the development of miniaturized and portable analytical devices based on microfluidic or lateral flow immunoassays for point-of-care applications and on the development of microscopy imaging methods for location and quantitative detection of antigens and target nucleic acid sequences in tissue sections and single cells. She is the co-author of more than 60 articles in international journals and a book chapters.

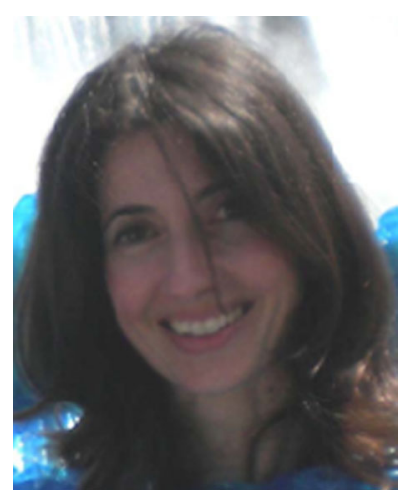

Elisa Michelini has been a researcher in analytical chemistry at the University of Bologna since 2006. Her scientific activity has been devoted to the development of bioanalytical methods including bioluminescent whole-cell biosensors and in-vivo assays with applications in drug screening, environmental monitoring, forensics, and diagnostics. Her main interests are in obtaining new bioluminescent proteins and the use of these to improve the analytical performance of cell-based assays and in-vivo imaging. More recently her activity has been devoted to the development of portable biosensing devices, for example chip-based whole-cell biosensors, for on-site multiplexed and point-of-need analysis. Since 2012 she has been a Member of the Scientific Advisory Board of the "International Society for Bioluminescence and Chemiluminescence" (ISBC). 